Service social

\title{
Schwartz, Mark (éd.), Using Computers in Clinical Practice : Psychotherapy and Mental Health Applications, New York, Haworth Press, 1984, 510 pages.
}

\section{Françoise Turgeon-Krawczuk}

Volume 36, numéro 1, 1987

L'informatique dans les services sociaux

URI : https://id.erudit.org/iderudit/706347ar

DOI : https://doi.org/10.7202/706347ar

Aller au sommaire du numéro

Éditeur(s)

École de service social de l'Université Laval

ISSN

1708-1734 (numérique)

Découvrir la revue

Citer ce compte rendu

Turgeon-Krawczuk, F. (1987). Compte rendu de [Schwartz, Mark (éd.), Using Computers in Clinical Practice : Psychotherapy and Mental Health Applications, New York, Haworth Press, 1984, 510 pages.] Service social, 36(1), 160-161.

https://doi.org/10.7202/706347ar d'utilisation que vous pouvez consulter en ligne.

https://apropos.erudit.org/fr/usagers/politique-dutilisation/ 


\section{RECENSIONS}

SChwartz, Mark (éd), Using Computers in Clinical Practice: Psychotherapy and Mental Health Applications, New York, Haworth Press, 1984, 510 pages.

Ce livre s'adresse aux cliniciens en santé mentale et aux administrateurs qui veulent savoir comment on peut utiliser les ordinateurs dans la pratique. Dans un langage accessible, il donne une vue générale et concise des différentes applications informatiques possibles dans les hôpitaux et cliniques psychiatriques et dans la pratique privée. Schwartz et ses nombreux collaborateurs sont, pour la plupart, psychiatres et psychologues.

Le livre se compose de soixante chapitres divisés en quatorze sections. Dans une introduction générale, les auteurs répondent à certaines questions préalables à l'introduction d'un système informatique, soit les conditions d'implantation et l'utilité. Une deuxième section porte sur l'impact de l'informatisation, les attitudes du personnel professionnel et clérical, les réactions et les résistances. Quatre parties plus brèves traitent du système de comptabilité, du traitement de texte, des tests et des rapports psychologiques. Dans les six sections suivantes, les auteurs abordent d'autres aspects cliniques touchant les applications de l'ordinateur dans la conduite d'entrevues, le diagnostic, le traitement, la réadaptation, la modification du comportement, l'apprentissage. Le livre se termine par deux sections plus générales portant sur le choix d'un ordinateur et sur quelques-unes de ses applications moins connues.

Comme dans la plupart des livres écrits en collaboration, les contributions sont inégales, certains articles étant meilleurs que d'autres. Même si l'ensemble est bien. structuré, aucun aspect n'est développé en profondeur et il n'y a pas d'unité dans la rédaction. Le livre reste facile à lire et contient nombre d'informations pratiques qui peuvent aider à éviter, ou du moins à anticiper, les frustrations qui accompagnent l'installation d'un système informatique.

Les aspects les plus intéressants pour un praticien en service social sont ceux qui touchent les activités directement reliées à la pratique professionnelle : évaluation, diagnostic, intervention auprès des clientèles. La moitié du livre environ rencontre cet intérêt spécifique et apporte des exemples d'applications informatiques à la pratique. En réponse au souhait généralisé des cliniciens de diminuer le temps consacré aux procédures évaluatives, plusieurs chapitres sont consacrés à la description de divers modèles d'admission assistés par ordinateur. On souligne que le système peut recueillir une partie de l'information directement auprès du client, faire l'analyse des réponses et soumettre sans délai au clinicien un résumé des résultats pertinents. Les auteurs tentent aussi de répondre à la question : la thérapie par ordinateur est-elle possible? Ils apportent plusieurs exemples d'applications informatiques qui supportent la thérapie, comme le jeu thérapeutique aidant à la 
modification du comportement ou l'enseignement individualisé aux personnes handicapées.

Il est certain que le développement des logiciels destinés à la pratique clinique ne répond pas encore aux attentes. Schwartz parle clairement des usages actuels et futurs des ordinateurs, mais aussi de leurs limites. Il livre quelques idées maîtresses : l'application technologique doit se faire sur mesure pour rencontrer les besoins spéciaux d'un organisme ou d'une clinique ; le succès d'un système dans un endroit ne garantit pas son succès ailleurs ; bien que les ordinateurs puissent régler certains problèmes, ils peuvent aussi en apporter de nouveaux; le développement et l'utilisation de cette technologie dans la pratique professionnelle sont beaucoup trop importants pour être laissés aux non-cliniciens.

La contribution spécifique du service social au livre de Schwartz est relativement peu importante. Sur soixante-cinq collaborateurs, une dizaine de travailleurs sociaux, gestionnaires ou professeurs, écrivent sur l'analyse des besoins de gestion, les attitudes du personnel face à l'informatique et les dossiers de placement dans un hôpital. Ils apportent très peu d'exemples d'applications cliniques en service social.

Il est essentiel que les travailleurs sociaux se familiarisent avec les utilisations actuelles des ordinateurs afin de pouvoir innover dans ce domaine. La lecture du livre de Schwartz nous ouvre des perspectives intéressantes en nous fournissant des modèles d'applications originales faites par d'autres cliniciens spécialistes de la relation d'aide.

Françoise TURGEON-KRAWCZUK

Hôpital Sainte-Justine,

Montréal.

Revue Computers in Human Services, éditée par Dick SCHOECH, New York, Haworth Press, vol. 1, no 1, 1985.

Les applications de nouvelles technologies dans les domaines des services sociaux et de la santé font l'objet de nombreuses publications aux États-Unis depuis le début des années 1970.

Il y a maintenant plus de vingt ans que se poursuit l'expérimentation des ordinateurs et de l'informatique dans les différents champs de l'intervention en sciences humaines tels que : l'éducation, la santé, la santé mentale, les services sociaux, etc. Et, jusqu'à récemment, ces publications paraissaient dans les revues spécialisées appartenant à ces différents champs. Depuis quelques années, les applications des technologies informatiques se sont développées à un point tel qu'une nouvelle revue se consacre maintenant exclusivement à ce sujet. II s'agit de la revue Computers in Human Services.

Devant initialement paraître quatre fois par année, le premier numéro date de 1985. Malheureusement, ses éditeurs accusent un retard important: le quatrième numéro de 1985 n'est paru qu'en 1987 ! Nous savons, par l'éditeur luimême, qu'il n'y aura aucune publication datée de 1986. La revue a cependant 